\title{
Diversité agromorphologique et commercialisation du gombo (Abelmoschus esculentus L.) à Ouagadougou et ses environs
}

\author{
Mahamadou SAWADOGO ${ }^{1 *}$, Didier BALMA ${ }^{2}$, Rasmata NANA ${ }^{1}$ et \\ Rodrigue METO-KAZILE TUOSAN LIVIUS SUMDA ${ }^{3}$ \\ ${ }^{1}$ Université de Ouagadougou (UFR-SVT) 09 BP 848 Ouagadougou 09 Burkina Faso. \\ ${ }^{2}$ INERA-CREAF/Kamboinse, 01 BP 476 Ouagadougou 01 Burkina Faso. \\ ${ }^{3}$ Institut Polytechnique Rural de Katiébougou, Mali. \\ *Auteur correspondant, E-mail : sawadogomahamadou@yahoo.fr, \\ sawma@univ-ouaga.bf; Tél. : +22670234587.
}

\section{RESUME}

Au Burkina Faso, on a observé récemment à Ouagadougou et ses environs une mobilisation de petits producteurs pour la culture du gombo destinée à la population urbaine. La présente étude entreprise sur la base d'enquêtes réalisées dans quatre localités environnant la ville de Ouagadougou et dans onze de ses marchés a permis de comprendre que la filière de production et de commercialisation du gombo frais procure des revenus substantiels (4846 FCFA/planche/producteur et $2241 \mathrm{FCFA} /$ jour/vendeur) aux différents acteurs malgré la précarité et l'absence d'une bonne organisation du circuit de commercialisation. Les maraîchers de Ouagadougou et de ses environs cultivent quatre variétés de gombo dont deux sont des variétés locales. Mais seule la variété hybride "Indiana" est beaucoup prisée par les acteurs grâce à sa précocité et à son bon rendement. Par ailleurs, les variétés locales sont également beaucoup appréciées pour leur forte teneur en mucilage, leur faible teneur en graines et pour leur saveur mais tendent à être délaissées pour leur cycle tardif et leur faible rendement. On note une forte demande des consommateurs pour l'amélioration des variétés traditionnelles et la disponibilité des fruits frais toute l'année. De même, sa mise en marché nécessite une réflexion sur son positionnement par rapport aux variétés traditionnelles, le conditionnement, les circuits de distribution et la promotion de la filière.

(C) 2009 International Formulae Group. All rights reserved.

Mots clés : Marché, Ouagadougou, revenue, maraîchers-culteurs, variétés, gombo.

\section{INTRODUCTION}

L'agriculture est la base du développement socio-économique du Burkina Faso mais elle est tributaire de multiples comportements qui entravent son épanouissement. Entre autres attitudes, on peut citer, le choix délibéré de nos structures de recherche et de développement de s'attacher aux céréales et au coton (MEDEV, 2005), laissant de côté d'autres produits agricoles recherchés sur le marché national, voire international pour leur apport considéré en éléments nutritifs (Agbo et al., 2008), à l'instar du gombo.

De plus en plus on assiste à un développement de la culture de gombo dont le marché national et international très actif, enregistre chaque année une hausse de la demande par rapport à l'offre. Ainsi de nos jours beaucoup sont de petits commerçants qui s'adonnent tant bien que mal à la commercialisation du gombo.

Cette culture reléguée au second plan (Adeniyi, 2001; Alabi et Esobhawan, 2006) 
sinon au dernier dans les différents programmes de recherche et de développement du Burkina Faso ne demande qu'un minimum d'investissements dans l'amélioration variétale et un apport technique pour générer des revenus additionnels aux producteurs et améliorer la valeur nutritionnelle des aliments pour les populations. Au Burkina Faso, le gombo est produit de manière générale pour la consommation familiale alors que les conditions agro-climatiques sont favorables pour sa production à grande échelle (Sawadogo et Balma, 2003). Cette situation est due au fait que l'espèce a toujours été considérée comme la culture de la femme dans le milieu traditionnel et a toujours servi simplement à la préparation des sauces à l'état frais ou transformé en poudre séchée (Sawadogo et al., 2006). Ce qui fait qu'on observe une absence de variétés performantes adaptées aux conditions agropédologiques (Ariyo, 2000) et aux exigences culinaires des populations, un manque de structures averties pour assurer l'écoulement du produit, une absence de motivation des producteurs pour accroître la production, un manque de semences de qualité, et la non maîtrise du circuit d'exportation.

Cette étude s'inscrit dans le cadre de la valorisation du gombo à travers un état des lieux de sa production au Burkina Faso pour une éventuelle amélioration qualitative de sa production, de son circuit de commercialisation et lui restituer sa place réelle dans le tissu agricole, commercial et même industriel. Pour ce faire, il faut chercher à comprendre les critères de choix des variétés de gombo cultivées autour de la ville de Ouagadougou et la plus value que cette culture apporte aux producteurs et aux commerçantes afin de proposer aux agriculteurs/maraîchers des variétés améliorées de gombo pouvant leur procurer des intérêts. Elle s'est donc fixé pour objectifs spécifiques d'inventorier les variétés cultivées par les maraîchers situés aux alentours de Ouagadougou, de faire une caractérisation morphologique des gombos cultivés et vendus dans les marchés de la plus grande agglomération du pays qu'est la ville de Ouagadougou afin de situer le goût des différents acteurs pour proposer aux producteurs des variétés améliorées créées au
Burkina Faso et d'établir un circuit d'approvisionnement des producteurs en semences de gombo rencontrant leurs intérêts.

\section{MATERIEL ET METHODE \\ Matériel végétal}

L'étude a porté sur les variétés de gombo cultivées directement dans les jardins maraîchers aux alentours de Ouagadougou, vendues sur la place du marché et rencontrées dans les différents points d'approvisionnement en semences de gombo. Il s'agit de deux (02) variétés commercialisées (Indiana et clemson) et deux (02) variétés locales (Man yanga et Gnu yega).

\section{Méthode}

L'étude a utilisé trois enquêtes, l'une auprès des maraîcher-culteurs de quatre localités de Ouagadougou et ses environnants, l'autre auprès des vendeuses ou commerçantes de gombo de onze marchés de Ouagadougou dont six construits et une troisième enquête a été réalisée au niveau des sources d'approvisionnement des jardiniers en semences. Un suivi de la production du gombo sur les parcelles des maraîcherculteurs a aussi été réalisé, suivi d'une caractérisation agromorphologique selon le descripteur de l'Institut International des Ressources Phytogénétiques (IPGRI). La dénomination des variétés par les producteurs a été considérée comme premier critère de distinction des variétés (Sadiki et al., 2007). A l'aide des fiches d'enquête, des interviews structurées et semi-structurées ont été réalisées (Sawadogo et al., 2005). Les résultats de celles-ci ont servi à orienter notre choix sur les variétés ayant servi à la caractérisation morphologique. $\mathrm{Au}$ total quinze (15) maraîcher-culteurs en moyenne ont été enquêtés par localité et quinze (15) vendeuses par marché, soit soixante quinze (75) maraîchers-culteurs et cent quatre vingts (180) vendeuses.

Les caractéristiques essentielles des localités et des marchés qui ont fait l'objet de cette étude sont présentées dans les tableaux $1 \mathrm{a}$ et $1 \mathrm{~b}$.

L'équipe de travail était composée d'enseignants-chercheurs de l'équipe d'amélioration du gombo de l'Université de Ouagadougou, de chercheurs de l'INERA, d'étudiants de l'Université de Ouagadougou et 
Tableau 1a: Les localités ayant fait l'objet des enquêtes.

\begin{tabular}{ll}
\hline \multicolumn{1}{c}{ Localités } & \multicolumn{1}{c}{ Localisation } \\
\hline Boulbi & Au Sud-Ouest de Ouagadougou, sur l'axe Ouaga-saponé, à 15 Km de \\
Boulmigou & Ouagadougou. \\
Koubri l'Ouest de Ouagadougou, sur l'axe ouaga-bobo \\
Loumbila & Sud-Ouest de Ouagadougou, sur l'axe ouaga-Kombissiri à 15 Km de \\
& Ouagadougou. \\
\end{tabular}

Tableau 1b: Les marchés ayant fait l'objet des enquêtes.

\begin{tabular}{llc}
\hline \multicolumn{1}{c}{ Marchés } & \multicolumn{1}{c}{ Localisation } & \multicolumn{1}{c}{$\begin{array}{c}\text { Nombre de } \\
\text { vendeurs enquêtés }\end{array}$} \\
\hline Nabi-yaaré & \multicolumn{1}{c}{ Mecteur 29, face hôpital saint Camille } & 15 \\
Pag-layiri & Secteur 15, sur l'avenue circulaire, après Ouga-inter & 18 \\
Zabre-daaga & Secteur 3, centre ville, côté Est de la BICIA-B siège & 15 \\
Kologo-naaba & Secteur 10, nord de Ouagadougou sur l'axe ouahigouya & 16 \\
Sankar-yaaré & Secteur 12, à côté de la cité “an III" & 18 \\
10-yaar & Secteur 10 de Ouagadougou, marché de Nonssin & 15 \\
\hline & \multicolumn{1}{c}{ Marchés non construits } & 14 \\
\hline Cité an II & Secteur 7, à la cité an II & 16 \\
Goughin & Secteur 8, sur la rue & 16 \\
Kar-palin & Secteur du côté du sallon International de l'Artisanat de & 15 \\
Tanghin & Ouagadougou (SIAO) & 15 \\
Dassassogo & Secteur 23, à côté du barrage de Tanghin & \\
\hline
\end{tabular}

de l'IPR/Katiébougou du Mali.

Le traitement statistique et les figures ont été réalisés avec le logiciel XL-STAT PRO.

\section{RESULTATS}

Les variétés cultivées et la variabilité agromorphologique

L'enquête menée auprès des maraîchers-culteurs exerçant dans les jardins des cinq localités sus-citées a révélé que quatre (4) variétés sont fréquemment rencontrées sur les parcelles de production : il s'agit de deux (2) variétés locales (Man yanga et Gnu yêga) et deux variétés importées (Indiana et Clemson). Parmi ces quatre variétés cultivées autour des retenues d'eau situées aux alentours de Ouagadougou, la variété hybride "Indiana" occupe la première place. En effet elle occupe en moyenne plus de $80 \%$ des superficies emblavées par le gombo (Figure 1).

L'analyse de variance des caractères quantitatifs des variétés (Tableau 2) montre que la différence entre la valeur maximale (max) et la valeur minimale (mini) est élevée pour le cycle $50 \%$ boutonnisation et floraison, pour le nombre de grains par capsule et le poids de mille grains. Les valeurs de l'écart type (E-type) montrent que la plupart des variables sont réparties autour de la moyenne (faible E-type). Néanmoins, on note une grande variabilité liée aux caractères 


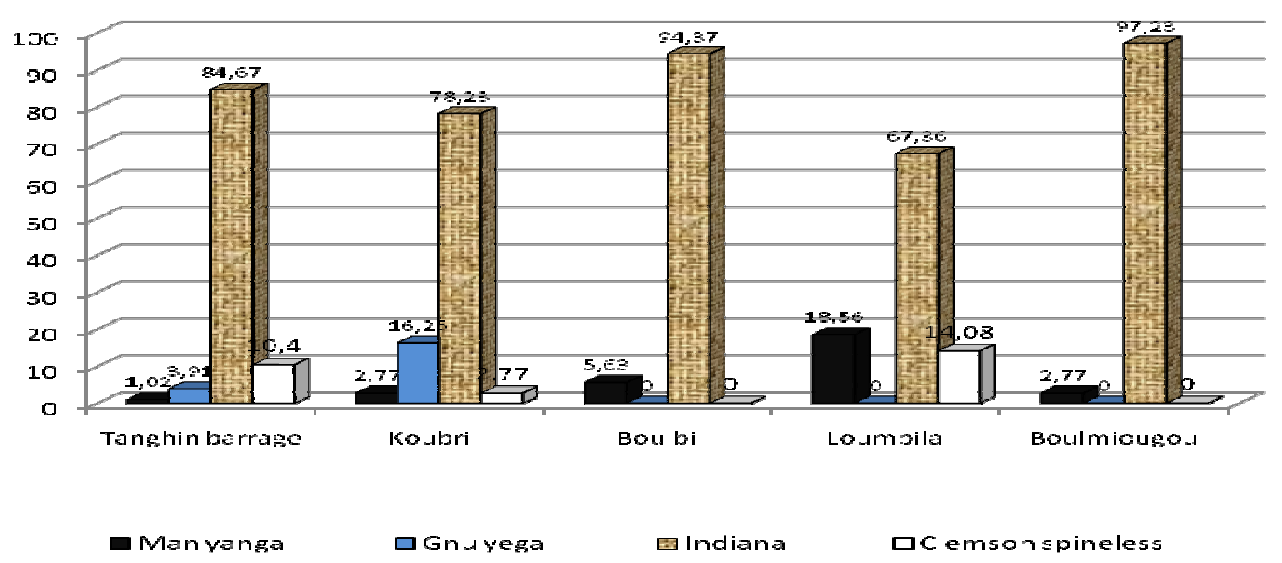

Figure 1: La répartition des variétés de gombo cultivées par les maraîchers-culteurs

Tableau 2 : Résultat de l'analyse de variance des caractères quantitatifs des variétés.

\begin{tabular}{|c|c|c|c|c|c|c|}
\hline Paramètres & Min & Max & Moyenne & E-Type & C.V. & $\begin{array}{c}\text { F observé } \\
(5 \%)\end{array}$ \\
\hline $50 \%$ Boutonnisation (jours) & 29,00 & 32,00 & 30,50 & 0,057 & 1,500 & $* *$ \\
\hline $50 \%$ Floraison (jours) & 47,00 & 52,00 & 49,75 & 0,041 & 1,785 & $* *$ \\
\hline Longueur capsule (cm) & 10,37 & 12,08 & 10,96 & 0,071 & 0,671 & $*$ \\
\hline largeur capsule $\mathrm{cm}$ ) & 1,15 & 2,16 & 1,88 & 0,259 & 0,422 & NS \\
\hline Nombre grains capsule & 26,50 & 46,17 & 38,24 & 0,248 & 8,209 & $* *$ \\
\hline Poids grains capsule (g) & 1,54 & 2,47 & 2,12 & 0,196 & 0,360 & $*$ \\
\hline Poids 1000 grains (g) & 42,30 & 52,04 & 47,38 & 0,099 & 4,079 & $* *$ \\
\hline
\end{tabular}

"nombre de grains par capsule et la largeur de la capsule". Cela est visible grâce aux valeurs élevées des CV. En effet, lorsque le coefficient de variation est élevé, cela traduit une grande variabilité liée au caractère étudié. A l'exception de la largeur de la capsule, toutes les variétés diffèrent significativement au seuil de $5 \%$.

La classification ascendante hiérarchique $(\mathrm{CAH})$ réalisée à partir des moyennes des variables quantitatives et avec comme paramètres la distance euclidienne et la moyenne des distances pondérées (Figure 2) montre avec une troncature au niveau 6 , une répartition des quatre variétés étudiées en trois groupes dont les deux premiers sont constitués chacun par une variété et le troisième par deux variétés (Clemson et Man yanga).

Selon les maraîchers-culteurs des différentes localités, la variété "Indiana" a un potentiel de production plus élevé que les variétés locales. Elle est essentiellement caractérisée par ses petites feuilles digitées, la coloration verte de toute sa partie végétative et de ses fruits (Tableau 3) mais aussi la précocité du cycle (les premières fleurs apparaissent à partir du $30^{\text {ème jour après }}$ semis). En outre, au champ, les plantes de cette variété forment un couvert végétal ouvert, facilement pénétrable, facilitant ainsi la cueillette.

Cependant, les variétés locales "man yanga", "gnu yêga" sont également rencontrées sur quelques parcelles mais avec une production très faible. En effet, les champs de ces variétés locales sont vite abandonnés puisque leur fructification est limitée dans le temps et elles possèdent des cycles tardifs. Les caractéristiques du fruit de 


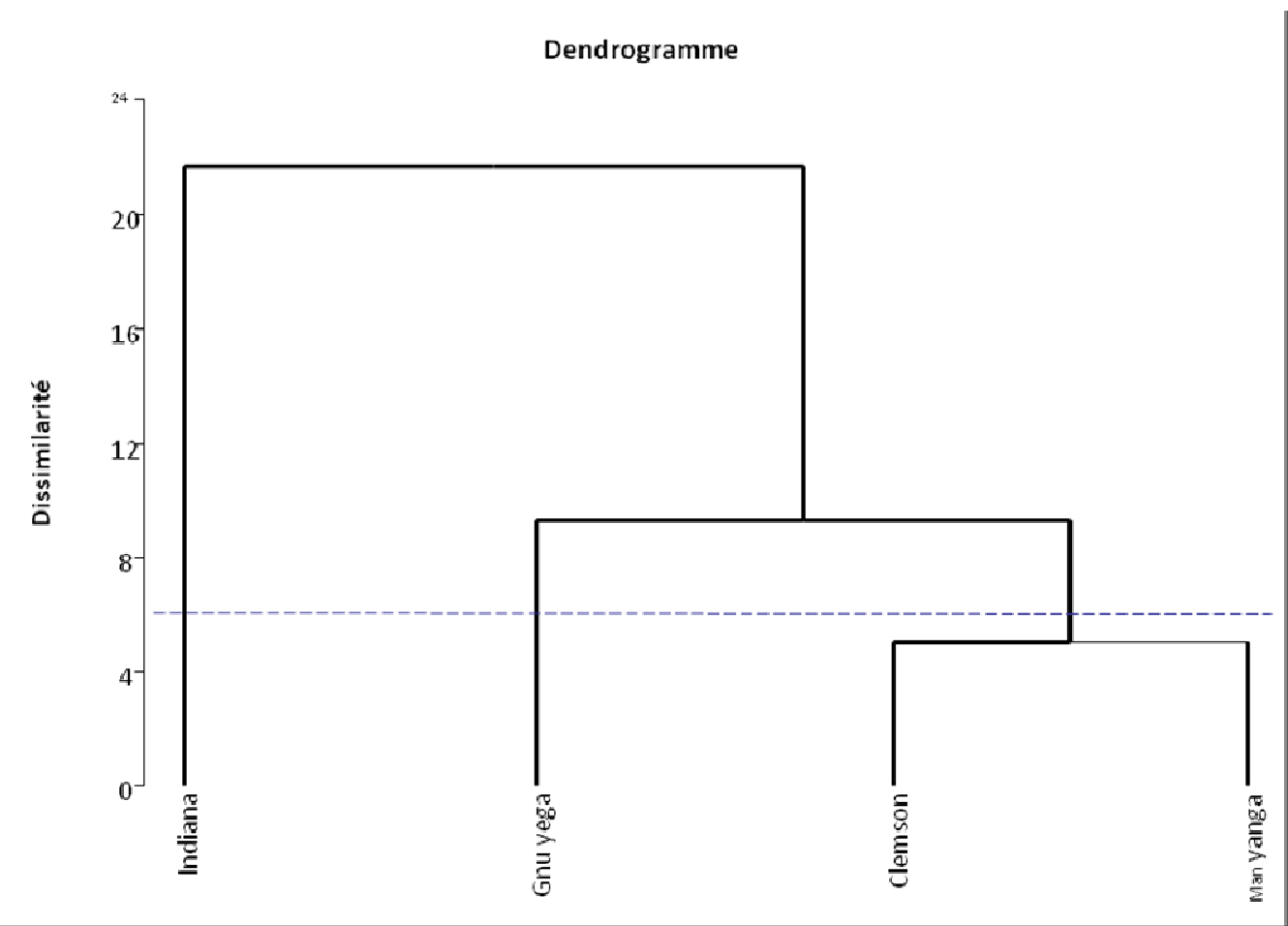

Figure 2: Dendrogramme de la classification hiérarchique ascendante.

la variété hybride "indiana" (Tableau 3) révèlent une homogénéité des caractéristiques du fruit au sein des différents marchés souvent approvisionnés par diverses sources de provenance.

\section{Le circuit semencier et la} commercialisation du gombo

Les enquêtes ont révélé l'existence d'un circuit semencier restreint opérant entre les maraîchers-culteurs et les vendeurs de semences. On a pu recenser près de quatre (4) à cinq (5) points de vente de semences (boutiques et magasins) par marché le plus proche de la localité enquêtée (Figure 3).

Ces points de vente constituent le lieu primordial d'approvisionnement des producteurs en semences. Les structures agréées pour la commercialisation des semences de gombo et intrants agricoles que sont l'Union des coopératives du Bam (UCOBAM) et King Agro sembleraient jouer les derniers rôles. Mais en réalité, les petits marchés des villages qui approvisionnent plus en semences ne sont que les points de relais de l'UCOBAM et de King Agro, qui sont les fournisseurs en gros de ces petits revendeurs. Les enquêtes réalisées dans les différents points d'approvisionnement en semences ont permis d'identifier en dehors des variétés Clemson spineless et Indiana, les variétés telles que Green star, Annie aotkley, Better five, Emerold green dont aucune trace n'a été retrouvée sur les parcelles des maraîchers-culteurs. Pourtant, les vendeurs de semences ont témoigné du bon rendement de celles-ci. Il faut dire que le circuit de semences améliorées de gombo est construit autour de l'UCOBAM et King Agro qui ne possèdent presque pas de parcelle-vitrine pour une meilleure visibilité des produits. Ce qui ne permet pas aux producteurs de faire un choix motivé et éclairé.

\section{La commercialisation du gombo et les gains}

Le gombo a longtemps été destiné à la consommation familiale en milieu rural mais depuis un certain temps, il est entré dans les habitudes alimentaires des citadins compte tenu de sa capacité d'entrer en combinaison avec d'autres légumes et légumineuses dans la composition des sauces de multiples plats. 
Tableau 3 : Caractéristiques essentielles du fruit de la variété hybride Indiana.

\begin{tabular}{|c|c|c|c|c|}
\hline \multirow[b]{2}{*}{ Marché } & \multicolumn{3}{|c|}{ Caractéristiques du fruit } & \multirow[b]{2}{*}{ FF } \\
\hline & $\mathbf{L}$ & D & $\mathbf{C F}$ & \\
\hline Sankar-yaaré (c) & $15,99 \pm 2,21$ & $1,47 \pm 0,12$ & Vert & Effilé \\
\hline Zabre daaga (c) & $13,91 \pm 1,02$ & $1,52 \pm 0,16$ & Vert & Effilé \\
\hline Pagla-yiri (c) & $15,65 \pm 1,52$ & $1,6 \pm 0,21$ & Vert & Effilé \\
\hline Dassasgo (o) & $13,41 \pm \mathbf{4 , 1 2}$ & $1,44 \pm 0,11$ & Vert & Effilé \\
\hline Gounghin (o) & $15,85 \pm 1,35$ & $1,45 \pm 0,08$ & Vert & Effilé \\
\hline Bendogo (o) & $14,55 \pm \mathbf{3 , 4 5}$ & $1,5 \pm 0,09$ & Vert & Effilé \\
\hline Baskuy (c) & $13,94 \pm 0,12$ & $1,58 \pm 0,01$ & Vert & Effilé \\
\hline 10 yaar $(\mathrm{c})$ & $15,63 \pm 1,07$ & $1,72 \pm 0,23$ & Vert & Effilé \\
\hline Nabi yaar (c) & $16,01 \pm 1,23$ & $1,37 \pm 0,15$ & Vert & Effilé \\
\hline Cité AnII (o) & $15,46 \pm 2,24$ & $1,54 \pm 0,26$ & Vert & Effilé \\
\hline Kaar palin (o) & $15,09 \pm 2,45$ & $1,49 \pm 0,31$ & Vert & Effilé \\
\hline Tanghin (o) & $15,34 \pm 1,78$ & $1,64 \pm 0,21$ & Vert & Effilé \\
\hline Moy & 15,06 & 1,52 & & \\
\hline E-Moy & 0,70 & 0,07 & & \\
\hline Var & 0,74 & 0,01 & & \\
\hline
\end{tabular}

Légende : L-longueur du fruit (cm); D- diamètre du fruit (cm); FF - forme du fruit; CF-couleur du fruit; (o) - marché ouvert; (c) - marché fermé.

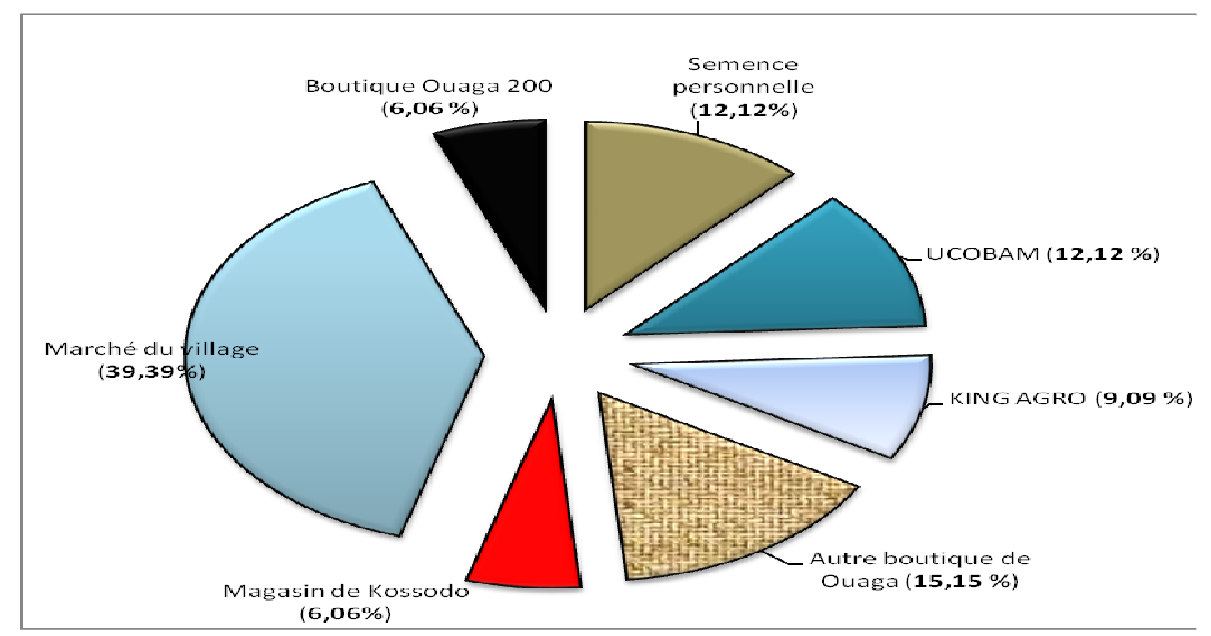

Figure 3: Lieu d'acquisition des semences par les maraîchers-culteurs.

En effet, il est fréquent de rencontrer des combinaisons telles que gombo-oseille, gombo-arachide, gombo-choux, gomboaubergine et autres. Cette utilisation multivariée a fait du gombo le légume le plus rencontré dans les sauces des citadins. Ainsi dans tous les onze (11) marchés enquêtés, le gombo est vendu tous les jours de l'année 
sous forme de fruits frais, de fruits séchés ou en poudre. Mais la forme fraîche est la plus désirée et appréciée par les vendeurs et les acheteurs.

Les tableaux 4 et 5 montrent qu'à des degrés divers les producteurs et les vendeurs gagnent de l'argent dans la production et la commercialisation du gombo. En effet, les producteurs des cinq (5) localités font un bénéfice net moyen de 4846,11 FCFA/ planche pendant que les vendeuses gagnent en moyenne 2241,13 FCFA par jour. Par ailleurs, si au niveau des producteurs il n'y a pas de grande disparité entre leurs gains, on en note une entre les vendeurs des différents marchés. Ainsi, les vendeuses des marchés de Zabre daaga, de Dassasgo, de 10 yaar et de la Cité an II font plus de bénéfices (de 3202 à 3450 FCFA/jour) dans la vente du gombo pendant que le gain journalier n'est que de 780,02 FCFA à Kaar palin et 938,96 FCFA à Bendogo.

Les résultats présentés (Figure 5) montrent que trois difficultés majeures ont été évoquées par les commerçants du gombo: il s'agit de l'inexistence de marché pour le gombo contrairement aux céréales et tubercules. Cette raison a été évoquée par toutes les commerçantes des marchés fermés à près de $85 \%$ des enquêtés et à environ 53-62\% des femmes des marchés ouverts (Figure 4). Ainsi le secteur de la commercialisation demeure précaire, archaïque et incertain.

Comme deuxième difficulté majeure, il faut retenir le manque de financement pour promouvoir et appuyer la filière de production et de commercialisation. A cela s'ajoute le taux élevé de fruits fibreux sur le marché.

\section{DISCUSSION}

La caractérisation agromorphologique

L'étude a montré qu'il existe peu de diversité variétale de gombo cultivé aux alentours de Ouagadougou alors que selon Sawadogo et Balma (2003) et Devra et al. (2007, 2008) les cultures agricoles telles que le gombo ont une diversité importante. Cette faiblesse de diversité s'explique par le fait de l'exigence des citadins quant aux

Tableau 4 : Gain journalier réalisé par les vendeurs des différents marchés.

\begin{tabular}{|c|c|c|c|c|c|}
\hline Marchés & QMFA & PAP (F CFA) & PV (F CFA) & DD (F CFA) & GM/V (FCFA) \\
\hline Sankar-yaaré (C) & 365 & 2423,64 & 4866,67 & 475 & 1968,03 \\
\hline Zabre daaga (C) & 558 & 3705,18 & 7440 & 500,2 & 3234,62 \\
\hline Pagla-yiri (C) & 490 & 3253,65 & 6533,33 & 600 & 2679,68 \\
\hline Dassasgo $(\mathrm{O})$ & 590 & 3917,66 & 7866,67 & 620 & 3329 \\
\hline Gounghin $(\mathrm{O})$ & 511 & 3393,09 & 6813,33 & 579 & 2841,24 \\
\hline Bendogo $(\mathrm{C})$ & 212 & 1407,7 & 2826,67 & 480 & 938,96 \\
\hline Baskuy (C) & 280 & 1859,23 & 3733,33 & 720 & 1154,1 \\
\hline 10 yaar $(\mathrm{O})$ & 580 & 3851,26 & 7733,33 & 680 & 3202,07 \\
\hline Nabi yaar $(\mathrm{C})$ & 390 & 2589,64 & 5200 & 483 & 2127,36 \\
\hline Cité AnII (O) & 900 & 7500 & 14625 & 3675 & 3450 \\
\hline Kaar palin $(\mathrm{O})$ & 189 & 1254,98 & 2520 & 485 & 780,02 \\
\hline Tanghin $(\mathrm{O})$ & 185 & 1228,42 & 2466,67 & 50 & 1188,25 \\
\hline Moyenne & 437,5 & 3032,04 & 6052,08 & 778,93 & 2241,11 \\
\hline $\mathrm{CV}$ & 0,49 & 0,59 & 0,57 & 1,20 & 0,47 \\
\hline $\begin{array}{l}\text { Ecart-type de la } \\
\text { moyenne }\end{array}$ & 66,27 & 544,71 & 1061,99 & 291,73 & 319,58 \\
\hline
\end{tabular}


Tableau 5 : Gain moyen réalisé par les producteurs/planche.

\begin{tabular}{|c|c|c|c|c|c|c|}
\hline Localisation & $\operatorname{SMC}\left(\mathbf{m}^{2}\right)$ & NMR & QGR & PVP (F CFA) & DD (F CFA) & GM/P (F CFA) \\
\hline Koubri & 511,12 & 21,75 & 1696,92 & 14140,99 & 10000,00 & 4140,99 \\
\hline Boulmiougou & 652,43 & 18,75 & 2166,07 & 18050,56 & 12355,00 & 5695,56 \\
\hline Loumbila & 750,11 & 24,50 & 2490,37 & 20753,04 & 16125,00 & 4628,04 \\
\hline Boulbi & 750,56 & 32,05 & 2491,86 & 20765,49 & 15500,00 & 5265,49 \\
\hline Tanghin & 650,62 & 28,21 & 2160,06 & 18000,49 & 13500,00 & 4500,49 \\
\hline Moyenne & 662,97 & 25,05 & 2201,05 & 18342,11 & 13496,00 & 4846,11 \\
\hline
\end{tabular}

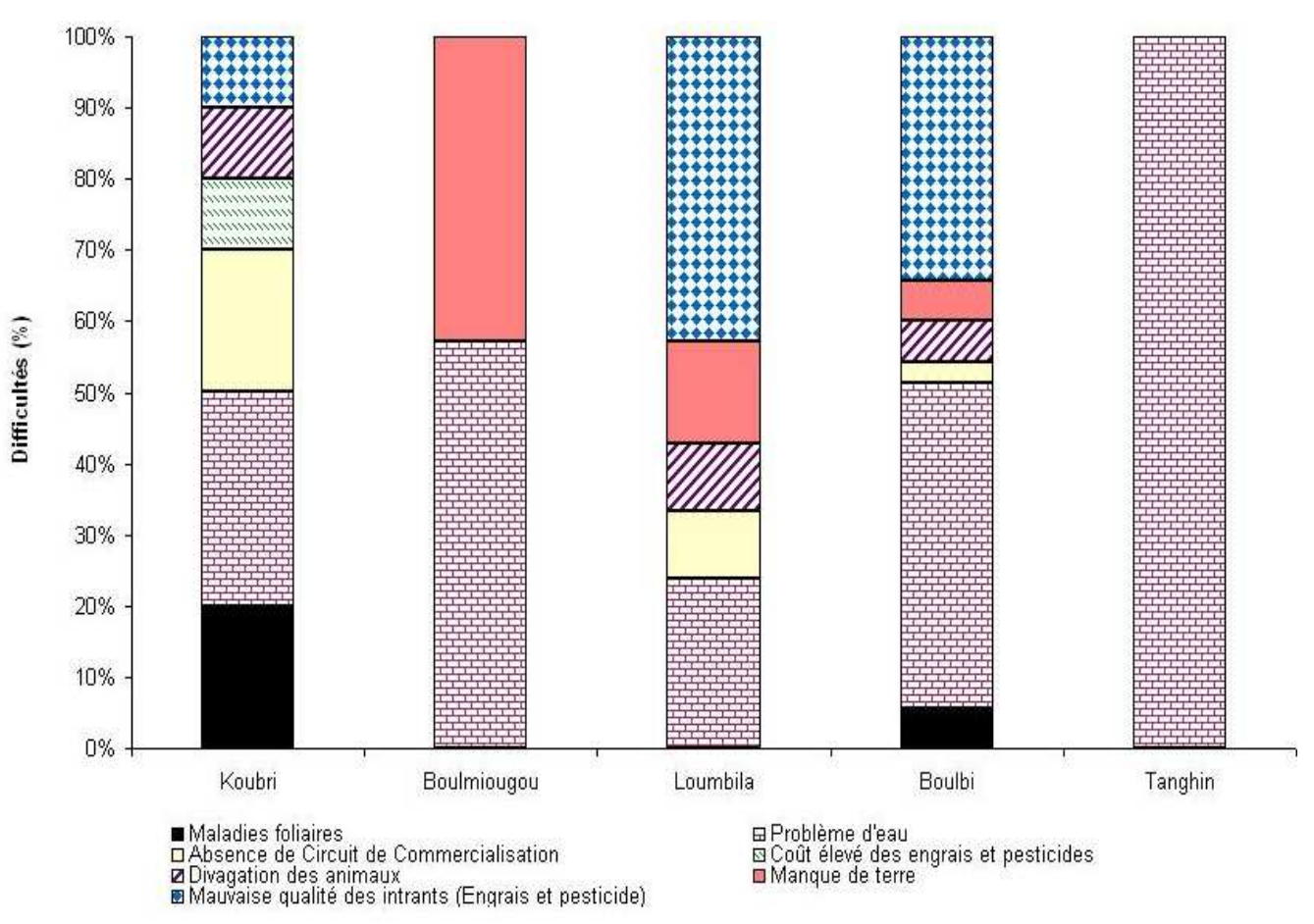

Figure 4 : Difficultés évoquées par les maraîchers-culteurs.

caractéristiques du fruit. En effet, le choix est plus orienté par le fruit que par tout autre critère. Ce sont les fruits de deux à trois jours de maturité après fécondation, ayant une longueur moyenne de $15,06 \mathrm{~cm}$ avec un diamètre moyen à la base du fruit de $1,52 \mathrm{~cm}$ qui sont vendus dans les onze marchés enquêtés. Ceci montre que le goût des consommateurs est orienté vers des fruits immatures et effilés. Selon Sawadogo et Balma (2003), les fruits immatures sont préférés par les consommateurs à cause de leur cuisson facile et de leur teneur élevée en mucilage. C'est pourquoi le gombo idéal pour la commercialisation est un fruit mesurant 4 à $9 \mathrm{~cm}$, vert, tendre et facile à écosser. Jusqu'à présent, le marché repose exclusivement sur la restauration. Néanmoins, il nous est très difficile de comprendre ou d'expliquer l'absence sur les parcelles des maraîchersculteurs. La seule hypothèse possible serait que ces variétés auraient perdu leur intérêt 
auprès des citadins et par conséquent elles seraient achetées par les producteurs pour être cultivées en milieu rural où elles sont encore acceptées. Ainsi, une caractérisation des deux types de variétés contribuerait à mieux comprendre les critères de préférence des variétés des citadins de ceux de la campagne. De plus, nous pensons que la création de variétés mieux adaptées à nos conditions agroécologiques et prenant en compte les critères de préférences des citadins contribueraient à une bonne promotion du gombo.

\section{La rentabilité financière de la culture du gombo}

Le gombo est suffisamment utilisé dans les campagnes et les centres urbains au point de susciter un intérêt économique certain. L'étude a montré une disparité de gain journalier par vendeur en fonction du marché qui est sans doute due à la localisation géographique du marché par rapport au centre ville de Ouagadougou, par rapport à la proximité des quartiers «Cité » qui regorgent de fonctionnaires ou de travailleurs salariés. Ainsi, les marchés de Zabre daaga et de la cité an II situés l'un au cœur de la ville de Ouaga aux environs des banques et des immeubles abritant l'administration et les sociétés privées, l'autre à l'intérieur de la cité an II sont les plus fréquentés par les consommateurs pour les achats du soir et du jour, permettant ainsi aux vendeurs d'améliorer le prix de vente et faire de gros bénéfices. Ce même constat avait été fait par les techniciens du Ministère de l'Economie et du Développement en 2005 (MEDEV, 2005). Ceci n'est pas le cas de Bendogo et Kaar palin qui en plus d'être situés à la périphérie de la ville sont des marchés ouverts, n'inspirant pas la confiance sanitaire de certains consommateurs. Mais de manière générale, le prix du gombo sur les différents marchés est beaucoup plus fonction de la situation géographique qu'à sa structuration (ouvert ou fermé).

On peut affirmer après notre étude que le circuit de commercialisation est naturellement vierge et nécessitera tout comme au niveau de la production, un travail technique accéléré surtout à cause des exigences liées à la production : notamment la certification, l'accréditation et le contrôle de normes qui constituent les aspects essentiels sur lesquels repose tout le processus commercial. Il faut aussi noter une forte autoconsommation en milieu rural.

En matière de commercialisation du gombo, il n'existe pas d'exigences particulières sur les marchés de Ouagadougou. $\mathrm{Ce}$ sont donc les habitudes commerciales appliquées aux produits frais qu'il convient de respecter. Néanmoins on peut citer entre autres l'emballage des fruits frais destinés aux supermarchés de la place tel que "Marina market". La commercialisation vise essentiellement le marché urbain. Pour la vente sur les marchés ouvert et fermé, les fruits sont mis en tas de 4-6 fruits à même le sol ou sur un plastique artisanal. Des circuits de distribution " spécialisés " (collecteurs, grossistes, semigrossistes, détaillants) se sont développés pour acheminer les produits des lieux de production en zone rurale vers les marchés urbains où ils sont le plus souvent vendus en " vrac ".

La mise en marché du gombo nécessite une réflexion sur le positionnement par rapport aux produits traditionnels, le conditionnement, les circuits de distribution et la promotion de la filière. Il est possible que les gombos améliorés ne puissent pas satisfaire l'ensemble des consommateurs actuels et qu'ils nécessitent une campagne de promotion mettant en avant les qualités nutritionnelles des fruits et des feuilles du gombo.

\section{Les difficultés de production et de commercialisation}

La production agricole de manière générale et du gombo frais en particulier durant toute l'année est fortement dépendante de la disponibilité en eau d'irrigation ou en retenues d'eau (barrages, forages, puits) fiables et permanentes toute l'année (Some et Ouattara, 2007). «Tant qu'il y a de l'eau pour arroser, le gombo Indiana n'arrête jamais sa production » a affirmé un producteur de Lumbila. En effet, des cinq localités enquêtées, c'est à Boulbi que la production du gombo par les hommes connaît une expansion. Cela serait dû à l'accessibilité en eau ; car à Lumbila, la culture du gombo est pratiquée presqu'exclusivement par les femmes alors qu'à Boulmiougou et à Boulbi, la production est dominée par les hommes 


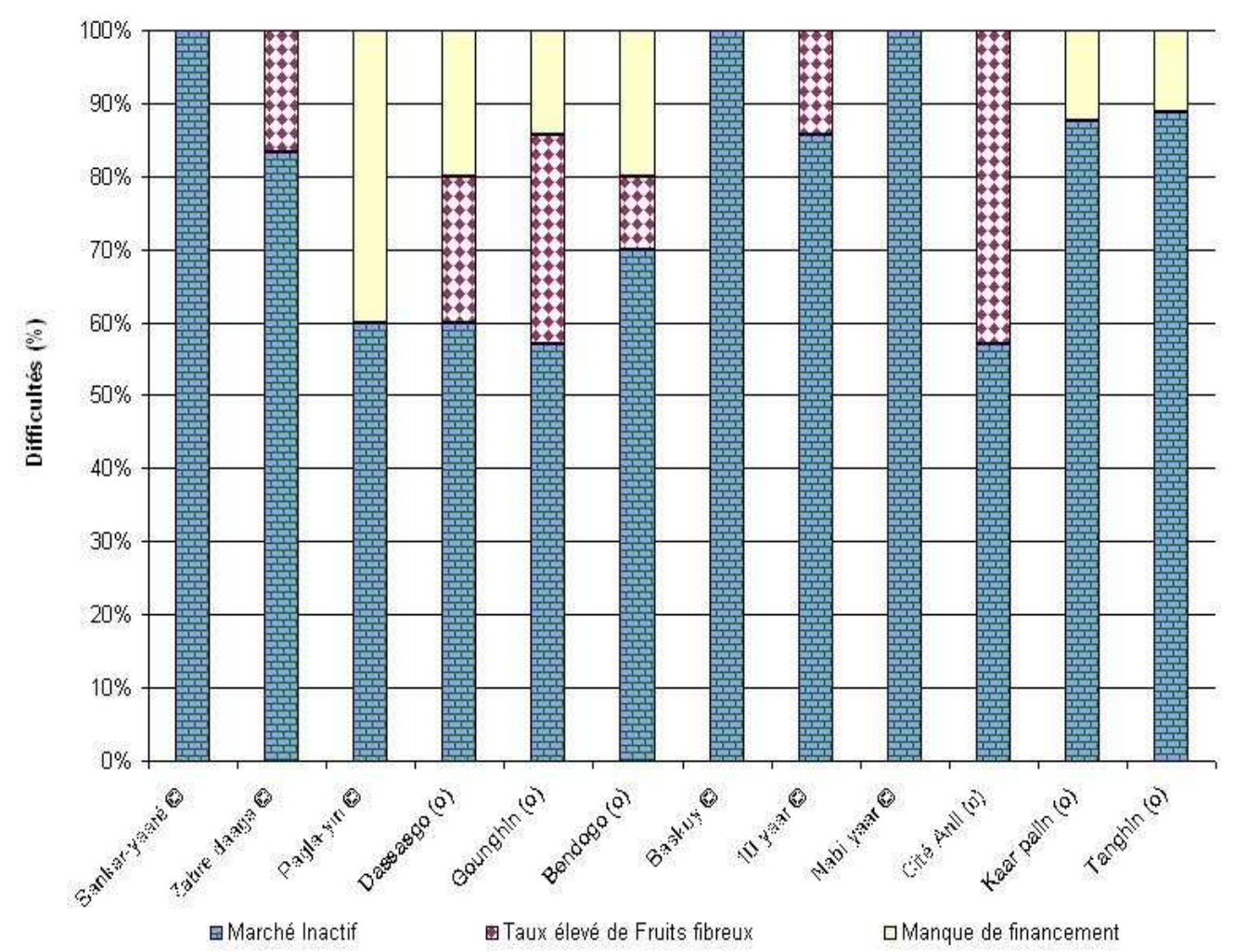

Figure 5: Difficultés évoquées par les vendeurs des marchés.

compte tenu de la pénurie d'eau dans les barrages contraignant les producteurs à creuser des puits pour arroser les plantes, tâche très ardue et onéreuse pour les femmes. En marge des marchés, les secteurs de la commercialisation du gombo au Burkina Faso demeurent très primitifs, composés en majorité par de très petits producteursrevendeurs qui écoulent tant bien que mal leurs produits à dos d'âne ou à vélo sur de petits marchés très souvent éphémères. Les fruits de gombo frais sont ainsi acheminés sans aucun soins sanitaire préalable, ni moyen de conservation, contraignant les vendeuses des marchés organisés à procéder à un conditionnement qui consiste seulement à un lavage des fruits et à les mettre dans des sachets de fortune.

Un financement adéquat aurait permis d'augmenter la production par l'extension des planches et des parcelles, permettant à chaque maraîcher-culteur ou à chaque groupement de producteurs de se doter d'une pompe à eau pour drainer de l'eau depuis le lit des barrages jusqu'aux parcelles de production.
Un autre problème limitant la qualité de la production du gombo demeure le mode de récolte. En effet, la récolte du gombo se fait à mains nues (sans gant) chaque deux ou trois jours par la cueillette des fruits immatures. Cette activité pénible et étalée dans le temps requiert une mobilité et une mobilisation de personnel très souvent onéreux pour les producteurs. Ceci entraîne fréquemment des cueillettes tardives, espacées de cinq à sept jours dont les produits devenus très fibreux réduisent la qualité du gombo et par conséquent son prix sur le marché.

\section{Conclusion}

La filière de production et de commercialisation du gombo frais est une activité qui procure des revenus substantiels aux différents acteurs malgré sa précarité et son manque d'organisation. Les maraîchersculteurs de Ouagadougou et ses environs cultivent quatre variétés dont deux sont des variétés locales. Mais seule la variété hybride Indiana est plus prisée par les acteurs à cause de sa précocité et de son meilleur rendement. 
Les variétés locales sont plus appréciées pour leur forte teneur en mucilage, leur faible teneur en graines et pour leur saveur mais délaissées dans le maraîchage pour leur cycle tardif et leur faible rendement.

Les études ont mis en évidence une demande des consommateurs pour l'amélioration des variétés traditionnelles, de la qualité de l'hygiène, la présentation et la disponibilité des fruits frais. On retrouve les mêmes demandes au niveau des circuits de distribution qui souhaitent également une amélioration de la conservation et une diversification de la production.

\section{BIBLIOGRAPHIE}

Adeniyi OR. 2001. An economic evaluation of intercropping tomato with okra in rainforest zone of Nigeria. The Journal of Horticulture Science and Biotechnology, 76(3): 347-349.

Agbo AE, Gnakri1D, Beugré GM, Fondio L, Kouamé C. 2008. Maturity degree of four Okra fruits varieties and their nutrients composition. Electronic Journal of Food and Plants Chemistry, 3(1): 1-4.

Alabi RA, Esobhawan AO. 2006. Relative economic value of maize - okra intercrops in rainforest zone, Nigeria. Journal of Central European Agriculture, 7(3): 433438.

Ariyo A. 2000. Analysis of genotype $\mathrm{x}$ environment interaction of okra (Abelmoschus esculentus L. Moench). J. Genet. Breed., 54(1): 35-40.

Devra IJ, Padoch C, Cooper HD. 2007. Managing Biodiversity in Agricultural Ecosystems. Published by Bioversity Intrenational, Columbia University press: New York; 492 p.

Devra IJ, Brown AHD, Cuong PH, ColladoPanduro L, Latournerie-Moreno L, Gyawali S, Tanto T, Sawadogo M, Mar I, Sadiki M, Hue NT-N, Arias-Reyes L, Balma D, Bajracharya J, Castillo F, Rijal D, Belqali L, Rana R, Saidi S, Ouedraogo J, Zangre R, Rhrib K, Chavez JL, Schoen D, Sthapit B, Paola de S, Fadda C, Hodgkin T. 2008. A global Perspective of the Richness and Evenness of Traditional Crop-variety Diversity Maintained by Farming Communities. PNAS Early Edition: The National Academy of Sciences of the USA; 1-6.

MEDEV (Ministère de l'Economie et du Développement). 2005. Profile des régions du Burkina Faso. Ministère de l'Economie et du Développement; 281p.

Ouattara K. 2007. Improved soil and water conservatory managements for cottonmaize rotation system in Western cotton area of Burkina Faso. Doctorate thesis, SLU, 25.

Sadiki M, Jarvis D, Rijal D, Bajracharya J, Hue NN, Camacho-villa TC, Burgos-May LA, Sawadogo M, Balma D, Lope D, Arios L, Mar I, Karamura D, Williams D, Chavez-Servia JL, Shapit B, Rao VR. 2007. An entry point of crop genetic diversity and distribution in agroecosystems. In Managing Bio-diversity in Agricultural Ecosystems. Bioversity International, Jarvis DI (ed). Colombia University press: Newyork; 34-76.

Sawadogo M, Balma D. 2003. Etude agromorphologique de quelques écotypes locaux de gombo cultivés au Burkina Faso. Science et Technique, Série Sciences Naturelles et Agronomie, 27(12): 111-129.

Sawadogo M, Ouédraogo J, Bélem M, Balma D, Dossou B, Jarvis D. 2005. Influence of ecosystem components on cultural practices affecting the in situ conservation of agricultural biodiversity. Plant Genetic Resources Newsletter, 141: 19-25.

Sawadogo M, Balma D, Zombré G. 2006. Expression de différents écotypes de gombo (Abelmoschus esculentus (L.)) au déficit hydrique intervenant pendant la boutonnisation et la floraison. BASE, Biotechnologie, Agronomie, Société, Environnement, 10(1): 43-54.

Some L, Ouattara K. 2005. Irrigation de complément et culture du sorgho au Burkina Faso. Agronomic Africaine XVII: 163-253. 